\title{
RADIATION AMPLIFICATION NEAR AN AUTOIONIZING STATE
}

\author{
P. LAMBropoUlos ${ }^{a, b}$, JIAN ZHANG ${ }^{a}$ AND S.J. VAN ENK ${ }^{a, b}$ \\ a Max-Planck Institut für Quantenoptik \\ Ludwig-Prandtl-Strasse 10,85748 Garching bei München, Germany \\ ${ }^{b}$ Foundation for Research and Technology-Hellas \\ Institute of Electronic Structure and Laser \\ P.O. Box 1527, Heraklion 71110, Crete, Greece \\ and Department of Physics, University of Crete, Greece
}

We present an internally consistent theory of amplification near the minimum of autoionizing states with quantitative application to calcium. We have included a completely specified three-photon pumping and we find certain unusual aspects of the system. We find conditions under which the system remains in a steady state, although being coupled to the continuum. For incoherent pumping we obtain quantitative results for the gain. Our results and their extensions are also discussed in the context of present-day experimental possibilities.

PACS numbers: $32.80 . \mathrm{Rm}, 32.70 . \mathrm{Jz}, 32.80 \mathrm{Wr}$

\section{Introduction}

During the last four years or so, questions pertaining to the coherent interaction of lasers with atomic or molecular states within the continuum have attracted considerable interest theoretically as well as experimentally. These problems may involve a smooth continuum or, as is more often the case, continua that are energetically degenerate with discrete states as for example in autoionization. Such discrete states embedded in continua with which they interact through intraatomic processes represent interesting structures which, on the one hand provide useful and unique insight into the details of intraatomic and intramolecular interactions, and on the other hand can be manipulated by means of external laser fields to produce new phenomena which are of relevance to a number of disciplines such as nonlinear optics, quantum optics, laser theory, etc. [1-5]. This possibility of coherent interaction within the continuum has led to a reconsideration of our traditional notions about transitions into the continuum. Under traditional weak-field radiation sources, a transition into the continuum is irreversible, it acts as a sink. But with coherent and moderately intense sources, it is possible to make transitions from the continuum back into bound states or from one bound state to another 
via the continuum. The presence of discrete states within the continuum, although not necessary, makes such processes much more probable. One class of phenomena related to these autoionizing states (AIS) concerns the possibility of radiation amplification without population inversion (AWoI) [6-11] which has been the subject of many discussions during the last four years. In pondering the problem of AWoI two aspects, namely an asymmetric absorption profile (or more generally an asymmetry between absorption and emission) and a mechanism for pumping population to the upper state, represent the cornerstones of any scheme. If an autoionizing state is to serve as the upper state, the asymmetric profile is provided by intraatomic interaction, but the pumping mechanism must be properly chosen. Since most often an autoionizing state cannot be modelled realistically by one discrete state interacting with a continuum, the question of pumping presents not only practical but also conceptual difficulties. The discrete state entering the formal model studies $[7,8]$ of AWoI through AIS is not a physical state, even if one discrete state coupled to a single continuum represents the atom adequately. In two recent papers $[12,13]$ we have identified some of the difficulties associated with multiphoton pumping of either autoionizing or autoionizing-like states. While most of the literature has concentrated on formal and qualitative aspects of AWoI, we have recently developed a theory of these phenomena that is quantitative in terms of the atomic structure and the interaction with a realistic pulse. We thus define a complete problem that includes pumping population into an autoionizing state by 3-photon absorption and amplification of an external probe beam. Assuming that the 3-photon pumping is incoherent and on resonance with the autoionizing state, and that the probe is tuned to the minimum of the autoionizing resonance, we obtain solutions describing the amplification process. We will give concrete qualitative applications to Ca. A more complex set of circumstances arises, however, if the energy of three pump photons is near or equal to the probe frequency. Then we have the coexistence of 3rd harmonic generation with the probe, which means that the harmonic can play the role of a probe. In that case, we would have the production of 3 rd harmonic through the internal process (but with some new features due to the presence of the AI state) and in addition its amplification through the population in the AI state. These two processes cannot be separated in an obvious way.

\section{The model}

For quantitative results we will consider a sequence of autoionizing doubly excited states of $\mathrm{Ca}$ belonging to the $3 \operatorname{dnp}\left({ }^{1} P_{1}\right)$ series which is also coupled to the series $3 d n f\left({ }^{1} P_{1}\right)$. These series $[14,15]$ decay by autoionization into the continuum $4 s \epsilon p$ and cannot be treated as isolated discrete states embedded in a continuum. To obtain the necessary atomic parameters, a separate calculation, which is a project in itself, must be performed. This has been accomplished in separate work [16], has been compared with available experimental data [17] on photoabsorption and has provided the parameters employed in this work. We assume that the atom is pumped from its ground state $4 s^{2}\left({ }^{1} S_{0}\right)$ to the vicinity of one of the ${ }^{1} P_{1}$ autoionizing resonances by a 3 -photon process. The required wavelength range for such pump- 
ing is around $565 \mathrm{~nm}$ which is readily accessible through presently available pulsed lasers. We consider in addition an external probe beam at frequency $\omega$ whose amplification or attenuation probes the existence or lack of positive gain under the specified pump conditions. After having determined the autoionizing spectrum and wave functions over an extended range of energy through techniques described elsewhere $[16,18]$, we consider an appropriately restricted energy range around the desired resonance. We fit the spectrum to the form $(q+\tilde{\delta})^{2} /\left(1+\tilde{\delta}^{2}\right)$, where $q$ will represent the Fano $q$ asymmetry parameter, and where the quantity $\tilde{\delta}$ expresses the detuning of the probe photon frequency $\omega$ from the transition frequency $|1\rangle \rightarrow|2\rangle$ in units of the autoionization width. Combined with the ab initio calculation of the transition to the continuum and the autoionization width, we can determine a set of parameters representing an effective discrete state plus continuum model which, however, contains all of the necessary complexity of the coupled-channel atomic problem.

\section{Dynamical evolution equations}

We consider a system consisting of an atomic medium, a probe field $\varepsilon$ and a pump field $\varepsilon_{\mathrm{P}}$. We assume that the pump field is externally imposed, with frequency $\omega_{\mathrm{P}}$ and wave vector $k_{\mathrm{P}}$. The complete dynamics of the system can then be described in terms of the atomic density matrix and of the complex amplitude of the probe field, both of them as a function of time and position. From this we can calculate all quantities needed in our problem as a function of pump frequency and intensity and of probe frequency. It is important to stress here that the equations do not imply pumping to a discrete part but a transition (pumping) involving the correct wave function at the energy $3 \omega_{\mathbf{p}}$. Extending formalism that we have employed in previous work $[8,19]$, we derive a set of equations for the reduced density matrix in a rotating frame with frequency $3 \omega_{P}$ and with the spatial factor $\exp \left(3 \mathrm{i} k_{\mathrm{P}} z\right)$ absorbed into its definition. We find the density matrix equations

$$
\begin{aligned}
& \left(\frac{\partial}{\partial t}+\gamma_{1}\right) \sigma_{11}=-2 \operatorname{Im}\left[\left(M_{21}^{(3)} \varepsilon_{\mathrm{P}}^{3}+M_{21} \varepsilon\right) \sigma_{12}\right] \\
& \left(\frac{\partial}{\partial t}+\Gamma_{2}\right) \sigma_{22}=2 \operatorname{Im}\left[\left(M_{21}^{*(3)} \varepsilon_{\mathrm{P}}^{3}+M_{21}^{*} \varepsilon\right) \sigma_{12}\right] \\
& \left(\frac{\partial}{\partial t}+\mathrm{i} \Delta_{12}+\frac{\gamma_{1}+\Gamma_{2}}{2}\right) \sigma_{12} \\
& \quad=-\mathrm{i}\left(M_{12}^{*(3)} \sigma_{22}-M_{12}^{(3)} \sigma_{11}\right) \varepsilon_{\mathrm{P}}^{* 3}-\mathrm{i}\left(M_{12}^{*} \sigma_{22}-M_{12} \sigma_{11}\right) \varepsilon^{*}
\end{aligned}
$$

Here

$$
M_{21}^{(3)}=M_{12}^{(3)} \equiv \sum_{l, m} \frac{\mu_{1 l} \mu_{l m}\left(\mu_{m 2}+\int \mathrm{d} \omega_{\mathrm{c}} \frac{\mu_{m c} V_{c 2}}{E_{m c}-\omega_{\mathrm{P}}}\right)}{\left(E_{1 l}-\omega_{\mathrm{P}}\right)\left(E_{1 m}-2 \omega_{\mathrm{P}}\right)} \equiv \bar{\mu}_{12}^{(3)}\left(1+\frac{\mathrm{i}}{\mathrm{q}_{3}}\right)
$$

is the effective 3-photon dipole transition moment between $|1\rangle$ and $|2\rangle$, where $\mu_{i j}$ denotes the usual electric dipole matrix element, and $V_{\mathrm{c} 2}$ the configuration interaction matrix element coupling $|2\rangle$ to the continuum. The integral contains a 
principal value part and an imaginary part at the pole. This matrix element is a very essential part of the pumping process containing the interference between the 3-photon transitions and autoionization, as expressed by the asymmetry parameter $q_{3}$. The one-photon dipole transition moment due to the probe of frequency $\omega$ is given by

$$
M_{21}=M_{12} \equiv \mu_{12}+\int \mathrm{d} \omega_{\mathrm{c}} \frac{\mu_{1 \mathrm{c}} V_{\mathrm{c} 2}}{\omega_{\mathrm{c}}-E_{1}-\omega} \equiv \bar{\mu}_{12}\left(1+\frac{\mathrm{i}}{\mathrm{q}}\right) \text {. }
$$

Furthermore, $\Delta_{12}=3 \omega_{\mathrm{P}}-E_{21}$, is the detuning of $3 \omega_{\mathrm{P}}$ from the 3-photon resonance between $|2\rangle$ and $|1\rangle$, with $E_{21}$ being the energy difference between $|2\rangle$ and $|1\rangle$, including the AC Stark shift of each state. The field-dependent ionization width $\gamma_{1}$ of $|1\rangle$ due to the absorption of pump or probe photons can be expressed as

$$
\gamma_{1}=\frac{4}{\Gamma_{2}}\left|\frac{\bar{\mu}_{21}^{(3)} \varepsilon_{\mathrm{P}}^{3}}{q_{3}}+\frac{\bar{\mu}_{21} \varepsilon}{q}\right|^{2},
$$

where $\Gamma_{2}=\pi\left|V_{\mathrm{c} 2}\right|^{2}$ is the autoionization width of $|2\rangle$. Finally we derive the wave equation in the slowly varying amplitude approximation for the amplitude $\varepsilon(z, t)$, after having removed the spatial factor $\exp \left(3 i k_{\mathrm{P}} z\right)$,

$$
\begin{aligned}
& \frac{\partial \varepsilon}{\partial z}+\frac{1}{c} \frac{\partial \varepsilon}{\partial t} \\
& \quad=\mathrm{i}\left(\Delta k-\frac{\delta}{c}\right) \varepsilon-\mathrm{i} \frac{\omega}{2 c \epsilon_{0}} N\left(M_{12}^{*} \sigma_{21}-\mathrm{i} \pi \mu_{1 \mathrm{c}} \mu_{\mathrm{cl}}^{(3)} \varepsilon_{\mathrm{P}}^{3} \sigma_{11}-i \pi\left|\mu_{1 \mathrm{c}}\right|^{2} \varepsilon \sigma_{11}\right),
\end{aligned}
$$

with $N$ the number of atoms. The phase mismatch $\Delta k$ is defined as $\Delta k=k-3 k_{\mathrm{P}}$, and similarly the detuning between pump and probe field is $\delta=\omega-3 \omega_{\mathrm{p}}$.

\subsection{Incoherent 3-photon pumping}

It turns out that the processes of harmonic generation and amplification cannot be separated if the pumping is coherent in the sense that the phase of the pump field relative to the probe field is well defined. In the case of incoherent pumping there is no such problem. Therefore, we consider here the latter case in order to study the amplification and to obtain an expression for the gain coefficient. The coherent case will be discussed elsewhere.

After a transient time of the order of $\Gamma_{2}^{-1}$, the coherence $\sigma_{12}$ has decayed to the value

$$
\sigma_{12}=\frac{-\mathrm{i}\left(M_{12}^{*} \sigma_{22}-M_{12} \sigma_{11}\right) \varepsilon}{\mathrm{i} \Gamma_{2} \tilde{\delta} / 2+\left(\Gamma_{2}+\gamma_{1}\right) / 2} .
$$

After this adiabatic elimination of the coherence, we can derive the following expression for the linear gain/loss coefficient for the probe, under the condition that there is no phase relationship maintained between the probe field and the pump field. That is, if the two fields are detuned with respect to each other, or in the case of phase mismatch, or for a phase-fluctuating pump field we obtain

$$
\kappa=\frac{N \omega}{2 \epsilon_{0} c n(\omega)}\left(\alpha_{1 T}^{\prime \prime} \sigma_{11}-\operatorname{Im}\left(M_{12}^{*} \sigma_{21}\right) / \varepsilon\right),
$$

where $\alpha_{1 T}^{\prime \prime} \equiv \pi\left|\mu_{1 \mathrm{c}}\right|_{E_{\mathrm{c}}=E_{1}+\omega}^{2}$, and $n(\omega)$ the index of refraction at $\omega$. 
Substituting $\sigma_{21}$ from Eq. (8) we finally obtain the gain coefficient

$$
\kappa=\frac{N \omega}{2 \epsilon_{0} c n} \frac{2\left(\bar{\mu}_{12}\right)^{2}}{\Gamma_{2}} \frac{\left[(\tilde{\delta}+q)^{2}+(\eta-1)\left(q^{2}+\eta\right)\right] \sigma_{11}-\eta\left(1+q^{2}\right) \sigma_{22}}{q^{2}\left(\tilde{\delta}^{2}+\eta^{2}\right)} .
$$

This result, which is one of our main formal results, shows that $\kappa$ depends explicitly on two important parameters: $q$ (lihe asymmetry parametcr of the resonance) and $\eta=1+\gamma_{1} / \Gamma_{2}$ (where $\gamma_{1}$ is the total ionization width of $|1\rangle$ directly into the continuum, and $\Gamma_{2}$ is the autoionization width). The gain $k$ can vary in time following the evolution of $\sigma_{11}$ and $\sigma_{22}$ under a pulse. It must be stressed here that the $\kappa$ in Eq. (9) is not the Raman gain but the gain of an amplifier due to the populations of the active medium, as clearly seen in Eq. (10). For $\eta=1$ and $q \rightarrow \infty$, Eq. (10) reduces to the usual gain which is proportional to the inversion $\sigma_{22}-\sigma_{11}$. For $\eta>1$ and $q<\infty$ we have the novel siluation created by the coherent interaction of a discrete state with a continuum.

\section{Results}

The solution of Eqs. (1)-(3) under prescribed pump and probe laser pulses provides the state of the atomic system as a function of time. We solved these equations numerically with a pump pulse whose time dependence is given by

$$
I(l)=I_{0} \operatorname{sech}^{2}(t / \tau)
$$

with $I$ being the intensity and $\tau$ what will be referred to as the pulse duration. Using the above expressions for the gain coefficient $\kappa$, we have explored the existence of positive gain as a function of pump pulse duration, frequency and intensity. Positive gain results only when the probe is tuned around the vicinity of the interference minimum of the autoionizing resonance [6-8]. Perhaps surprisingly, a broad resonance with small $q$ value may produce much more gain than a narrower one with larger $q$. This we have traced to the difference in 3-photon ionization directly into the $f$ continuum which does not enter in the interference channels but represents an independent incoherent loss (of atomic population) mechanism. This is documented below with a specific example of two resonances. In general, we have found that both the maximum gain and the frequency range of positive gain increase with increasing pump intensity, up to a point, beyond which the medium is depleted because of ionization. A quantitative assessment of the above findings is provided in Figs. 1 and 2. Figure 1 demonstrates the dependence of the averaged gain per pulse on intensity for three different pulse durations for the states $3 d 5 p\left({ }^{1} P_{1}\right)$ and $3 d 6 p\left({ }^{1} P_{1}\right)$ whose autoionization widths are $634 \mathrm{~cm}^{-1}$ and $35 \mathrm{~cm}^{-1}$, respectively. As mentioned above, we have here a clear case where the narrow state produces gain three orders of magnitude smaller than the much broader one. In Fig. 2, we present the temporal behavior of four key quantities: gain, populations of $|2\rangle$ and $|1\rangle$ and ionization, for different intensities. First, we note an increase in the gain with the pump intensity. Second, we note that in Fig. 2c during part of the pulse (from about $-0.9 \tau$ to $+0.9 \tau$ ) we have inverted populations $\left(\sigma_{22}>\sigma_{11}\right)$, while otherwise we have $\sigma_{22}<\sigma_{11}$. We have therefore 

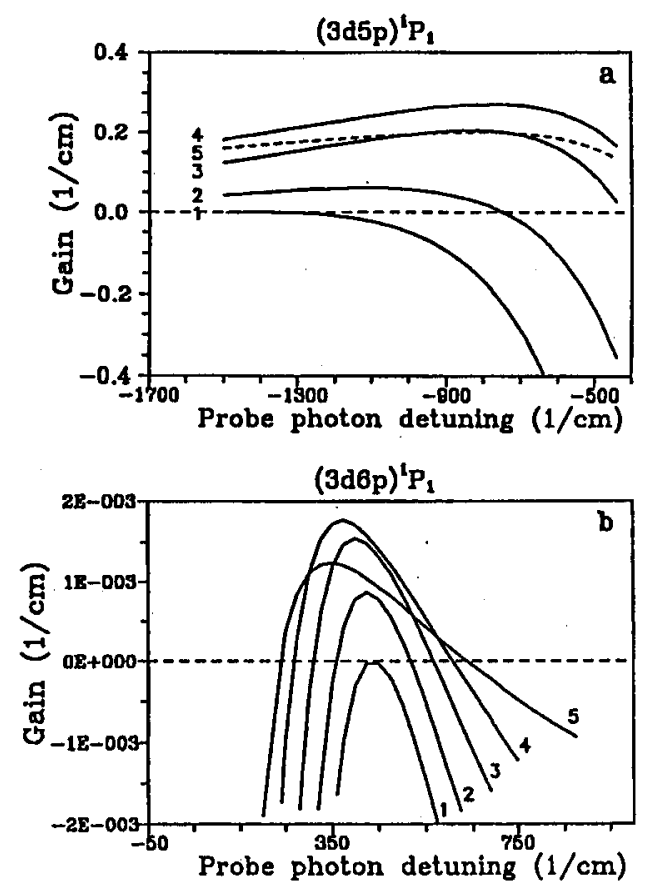

Fig. 1. Averaged gain over a 1 ps pulse as a function of the detuning for (a) $(3 d 5 p){ }^{1} P_{1}$ as upper state with different pump laser intensities: curve (1) $0 \mathrm{~W} / \mathrm{cm}^{2}$, (2) $3 \times 10^{11}$ $\mathrm{W} / \mathrm{cm}^{2}$, (3) $4 \times 10^{11} \mathrm{~W} / \mathrm{cm}^{2}$, (4) $5 \times 10^{11} \mathrm{~W} / \mathrm{cm}^{2}$, and (5) $7 \times 10^{11} \mathrm{~W} / \mathrm{cm}^{2}$; (b) $(3 d 6 p){ }^{1} P_{1}$ as upper state with different pump laser intensities: curve (1) $0 \mathrm{~W} / \mathrm{cm}^{2},(2) 5 \times 10^{10}$ $\mathrm{W} / \mathrm{cm}^{2},(3) 7 \times 10^{10} \mathrm{~W} / \mathrm{cm}^{2}$, (4) $1 \times 10^{11} \mathrm{~W} / \mathrm{cm}^{2}$, and (5) $2 \times 10^{11} \mathrm{~W} / \mathrm{cm}^{2}$.

amplification without inversion, but surprisingly under certain conditions we also liave inversion. This is rather unexpected since our pumping occurs through an electromagnetic transition, but it can be explained by the phenomenon of a nondecaying state.

\section{Nondecaying state}

Here we discuss briefly a fundamental and rather counterintuitive leature of an autoionizing state excited by a single or even by more than one field. Namely, it can reach a steady state although the system is connected to the continuum. This phenomenon (whose origins are implicit in Refs. [1] and [2]) plays a role in the behavior seen in Fig. $2 \mathrm{~b}$ where ionization and populations stabilize at the end of the pulse. We can show that if the probe field is sufficiently weak, then the atomic system will reach a steady state within a few $\Gamma_{2}^{-1}$, provided that the following relation holds between the detuning and the ionization rates:

$$
\Delta_{12}=-q_{3}\left(\Gamma_{2}-\gamma_{1}\right) / 2
$$

The relative steady-state populations in the autoionizing and lower states are, 

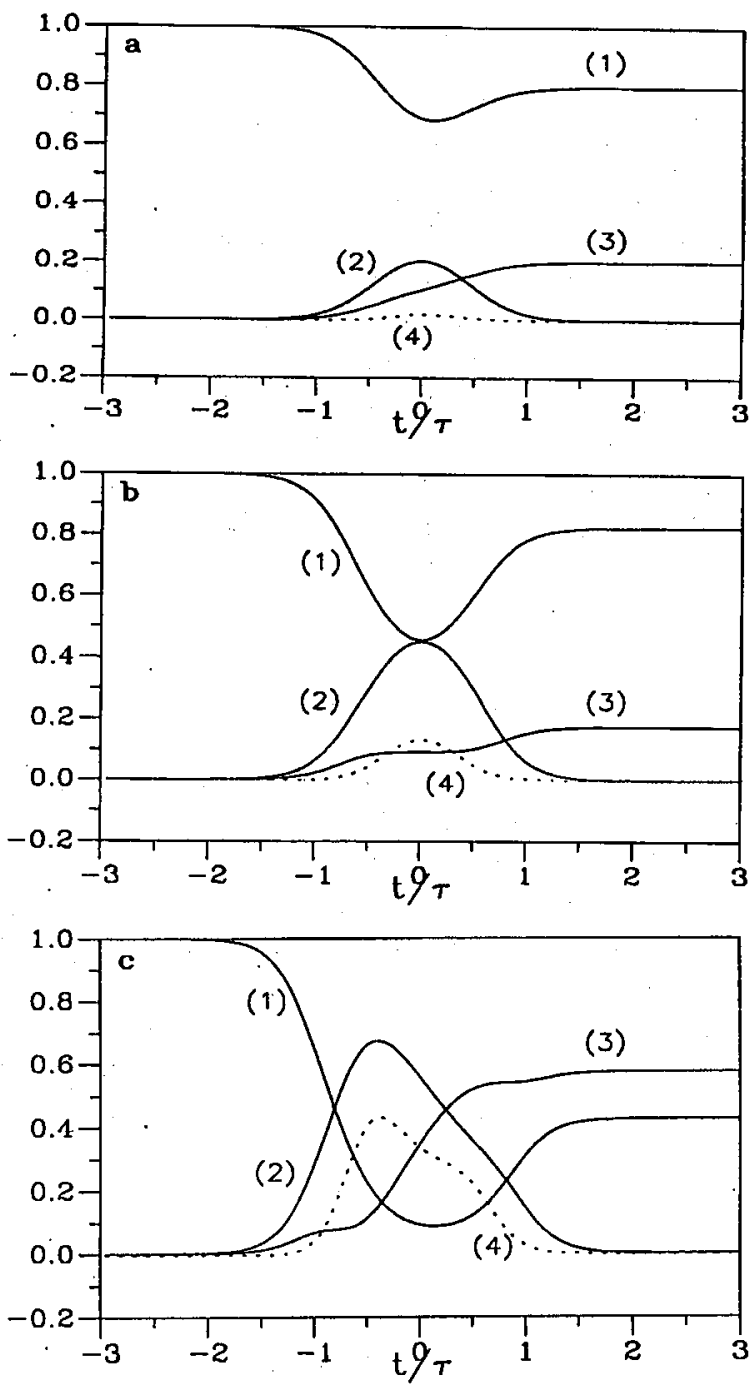

Fig. 2. Time-dependent behavior of (1) ground state population, (2) upper state population, (3) ionization and (4) $\kappa\left[\mathrm{cm}^{-1}\right]$ in the case of $(3 d 5 p){ }^{1} P_{1}$ as upper state for a 1 ps pulse at 3 different pump laser intensities: (a) $2.0 \times 10^{11} \mathrm{~W} / \mathrm{cm}^{2}$, (b) $3.0 \times 10^{11}$ $\mathrm{W} / \mathrm{cm}^{2}$ and (c) $5.5 \times 10^{11} \mathrm{~W} / \mathrm{cm}^{2}$.

respectively,

$$
p_{2}=\frac{\gamma_{1}}{\Gamma_{2}+\gamma_{1}}, \quad p_{1}=\frac{\Gamma_{2}}{\Gamma_{2}+\gamma_{1}} .
$$

Thus if the system is pumped sufficiently hard at the appropriate detuning given by (12) (such that the ionization rate $\gamma_{1}$ from the lower state exceeds the ionization rate $\Gamma_{2}$ from the upper state), inversion between the two populations will be cre- 
ated. The system, however, does not decay because of the simultancous destructive interference between the two different paths of ionization for both the autoionizing and the lower state. This steady state is transparent to the probe field: the system neither absorbs nor cmits photons by stimulated processes, so that there is no amplification. Away from this nondecaying state there is amplification, but the system in that case decays slowly, the decay rate depending on how far the detuning $\Delta_{12}$ is from the value given by (12). When the system is pumped by a pulse, the intensity of the pump field varies in time. At a fixed detuning, therefore, the atoms will be driven towards the exact stcady state only at certain times, namely when $\gamma_{1}$ satisfies the condition (12). Otherwise, the system will slowly or rapidly decay, but will in the process display amplification. This is what one sees in Fig. 2. If the probe field becomes stronger, then in general the steady state will disappear and the system will be ionized. Only if the relative pliase between the probe and the pump field equals either 0 or $\pi$ can the steady state continue to exist. The detuning has to satisfy a condition diflerent from (12), but the populations in the nondecaying state are still given by (13).

\section{Conclusions}

Within the framework of an internally consistent formalism, we have shown that multiphoton (colierent or incoherent) pumping can provide a realistic scheme of amplification with or without inversion near the minimum of an AIS. On the basis of realistic atomic parameters and a time-dependent analysis we have obtained the gain for a probe pulse of an appropriate duration. For the calculation of the gain, we have assumed an atomic density of $10^{17} \mathrm{~cm}^{-3}$ which can be obtained in a heatpipe. Our intention here is not to provide a working setup for a practical amplifier but a realistic situation for testing the validity of the fundamentals of the model with presently accessible cxperimental means. The overall accuracy of our atomic model (as tested by comparison with whatever experimental or theoretical data are available) provides we believe sufficient confidence to not deter us from proposing this (or a closely related scheme) for immediate experimental study. The usual question of whether a basis exists in which the populations of $|2\rangle$ and $|1\rangle$ are inverted is here irrelevant because there is no dressing by external fields. The asymmetry is provided by intraatomic interactions which determine the only physically meaningful basis. In any case, we prefer to have the cmphasis on the novelty of these amplification (and eventually lasing) schemes, with reduced requirements on inversion, rather than on the complete absence of inversion. Experimental realization of our scheme in a medium of density $10^{17} \mathrm{~cm}^{-3}$ may also lead to the generation of the 3rd harmonic of the pump photon. This harmonic generation is appreciable if the system is close to a steady state, where the populations in both states, although coupled to the continuum, do not decay. This radiation may serve as the radiation to be amplified through the mechanism of this paper. We have here chosen the probe as a separate incolicrent external source in order to concentrate on the basic mechanism of pumping and amplification. We will discuss the problem with the 3rd harmonic included in a separate publication. Experimentally one can always decouple it from the amplification through phase matching and/or 
detuning the pump away from the minimum, as has been the case here in the quantitative and numcrical results.

\section{Acknowledgment}

The work of S.J.v.E. is supported by the IIuman Capital and Mobility programme under contract No. ERBCIIBGCT920192.

\section{References}

[1] P. Lambropoulos, P. Zoller, Phys. Rev. A 24, 379 (1981).

[2] J.II. Eberly, K. Rzązewski, D. Agassi, Phys. Rev. Lelt. 49, 693 (1982); Z. Deng, J.II. Eberly, J. Opt. Soc. Am. B 1, 102 (1984).

[3] H. Bachau, P. Lambropoulos, Z. Phys. D 11, 37 (1989); H. Bachau, P. Lambropoulos, R. Shakeshaft, Phys. Rev. A 34, 4785 (1986).

[4] X. Tang, Z. Phys. D 6, 255 (1987).

[5] T. Nakajima, P. Lambropoulos, Phys. Rev. Letl. 70, 1081 (1993).

[6] V.G. Arkhipkin, Yu.I. IIeller, Phys. Lett. A 98, 12 (1983).

[7] S.E. IIarris, Phys. Rev. Lett. 62, 1033 (1989).

[8] A. Lyras, X. Tang, P. Lambropoulos, Jian Zhang, Phys. Rev. A 40, 4131 (1989).

[9] D. Kupiszewska, P. Masiak, K. Rzążewski, Opt. Commun. 92, 266 (1992).

[10] O. Kocharovskaya, Phys. Rcp. 219, 175 (1992), and references therein.

[11] A. Nottelmann, C. Peters, W. Lange, Phys. Rev. Lett. 70, 1783 (1993).

[12] H. Bachau, A. Lyras, P. Lambropoulos, Opt. Commun. 83, 331 (1991).

[13] A. Lyras, X. Tang, P. Lambropoulos, Opt. Commun. 92, 355 (1992).

[14] C.H. Greene, L. Kim, Pliys. Rev. A 36, 2706 (1987).

[15] K. Ueda, Phys. Rev. A 35, 2484 (1987).

[16] Jian Zhang, Ph.D. Thesis, University of Southern California, unpublished; P. Lambropoulos, Jian Zhang, X. Tang, Coherence Phenomena in Atoms and Molecules in Laser Field, Plenum Press, New York 1992.

[17] G.H. Newsom, Proc. Phys. Soc. 87, 975 (1966); V.L. Carter, R.D. Hudson, E.L. Brieg, Phys. Rev. A 4, 821 (1971).

[18] T.N. Chang, Phys. Rev. A 39, 4946 (1989).

[19] Jian Zhang, P. Lambropoulos, Phys. Rev. A 45, 489 (1992). 